\title{
Assessment of intervention outcome in the absence of baseline data: 'reconstruction' of condom use time trends using retrospective analysis of survey data
}

\author{
C M Lowndes, ${ }^{1,2}$ M Alary, ${ }^{2}$ S Verma, ${ }^{3}$ E Demers, ${ }^{2} \mathrm{~J}$ Bradley, ${ }^{3}$ A A Jayachandran, ${ }^{3}$ \\ B M Ramesh, ${ }^{4}$ S Moses, ${ }^{5}$ R Adhikary, ${ }^{6}$ M K Mainkar ${ }^{7}$
}

- Supplementary figures/ tables/appendix are published online only at http://sti.bmj.com/ content/vol86/issue1

1 Department of HIV \& Sexually Transmitted Infections, Health Protection Agency, London, UK ${ }^{2}$ URESP, Centre de recherche FRSO du CHA universitaire de Québec, Québec, Canada ${ }^{3}$ CHARME-India Project, Bangalore, India ${ }^{4}$ Karnataka Health Promotion Trust, Bangalore, India ${ }^{5}$ Department of Medical Microbiology, University of Manitoba, Winnipeg, Canada ${ }^{6}$ Family Health International, New Delhi, India ${ }^{7}$ National AIDS Research Institute, Pune, India

\section{Correspondence to}

Dr Catherine Lowndes,

Department HIV \& STIs, Centre for Infections, Health Protection Agency, 61 Colindale Avenue, London NW9 5EQ, UK; catherine.lowndes@hpa.org.uk

Accepted 1 December 2009

This paper is freely available online under the BMJ Journals unlocked scheme, see http://sti. bmi.com/site/about/unlocked. xhtml.

\section{ABSTRACT}

Background Avahan, the India AIDS Initiative, is a large-scale targeted intervention. Data on condom use prior to Avahan are unavailable. The authors used a novel method of analysing cross-sectional survey data to 'reconstruct' condom use rates in presurvey years, and to assess the relationship between Avahan and time trends in condom use among female sex workers (FSWs). Methods Among FSWs reporting consistent condom use (CCU) with clients in surveys from 21 districts in southern India $(n=7358)$, data on length of time using condoms, and length of time selling sex, were converted into number of FSWs using condoms (numerator) and selling sex (denominator) by year, to give yearly rates of CCU from 2001 to year of survey. Linear regression with generalised estimating equations was used to assess time trends and to compare the rate of increase in condom use before and after Avahan initiation in 2003. Results In all 21 districts, the rate of increase in CCU from 2001 to time of the surveys was highly significant. Overall CCU increased from $27 \%(22 \%)$ with occasional (regular) clients in 2001 , to $76 \%(68 \%)$ in 2006 , respectively. The yearly rate of increase in CCU was significantly greater after (slope 2003-2006: 12.7\% $(12.0 \%)$ per year for occasional (regular) clients) than prior to Avahan (slope 2001-2003: 5.1\% (5.3\%) per year for occasional (regular) clients) implementation $(\mathrm{p}<0.0001)$.

Conclusions The findings indicate a positive relationship between implementation of the Avahan programme and rates of CCU increase among FSW. This method of analysis may be useful in other contexts where preintervention data are lacking.

\section{INTRODUCTION}

Avahan, the India AIDS Initiative of the Bill \& Melinda Gates Foundation, is a large HIV prevention programme targeting female sex workers, their clients, men who have sex with men and injectiondrug users in the six Indian states most affected by HIV. ${ }^{1}$ The evaluation framework for the programme includes extensive biological and behavioural data collection among targeted groups and the general population, in combination with transmission dynamics modelling to estimate intervention impact (infections averted) and cost-effectiveness. ${ }^{2} 3$

One of the main outcome indicators of the Avahan programme is trends in condom use by female sex workers (FSWs) with their clients prior to and following implementation of the Avahan intervention. The main method for assessing trends in condom use over time in the project is using data from face-to-face interviews with FSWs carried out as part of serial cross-sectional surveys termed integrated biological and behavioural assessments (IBBA). ${ }^{4}$ Accurate data on pre- and baseline condom use rates are also essential for the mathematical modelling component of the evaluation. ${ }^{3} 56$

However, due to the timing of the cross-sectional IBBA surveys in relation to intervention start times, with the surveys generally being carried out 1-2 years after intervention start, there is a lack of baseline data on condom use rates among the target populations. There is also a lack of comparable data on condom use rates prior to the intervention: even in states/districts where data from pre-Avahan surveys among sex workers are available, in general, the unit of analysis/possible breakdown (district/state) and/or sampling strategy and frame are not comparable with those used within Avahan, or there is insufficient information available to assess whether or not the samples are comparable. ${ }^{4}$

This lack of preintervention/baseline data is a problem in many contexts, since it is difficult to undertake surveys which collect sensitive behavioural and biological information from stigmatised populations until programmes which benefit these populations are established, which takes time. ${ }^{47}$ Monitoring data from the Avahan programme indicate that it took 5 years to achieve a high and frequent coverage rate of the target population of FSW, with approximately $10 \%$ of the estimated target population contacted monthly by a peer educator after 1 year, $50 \%$ after 3 years and $75 \%$ after 5 years. ${ }^{8}$ Similarly, another targeted intervention programme initiated in Gujarat in 1997 had reached approximately 400 FSW within the first year and, after a further 5 years, was in contact with over 3000 FSW. $^{9}$ Such data indicate that collection of comparable preintervention/baseline data, and indeed even definition of programme start point, are challenging in the context of time trend evaluations of intervention impact among mobile, stigmatised populations.

Since the first round of IBBA was carried out in most districts $\sim 1-2$ years after the start of the intervention, condom use rates measured in the IBBA may well have increased since the start of the programme, and thus would be higher than at baseline. In order to enable assessment of the relationship between programme implementation and behavioural change, and for accurate impact assessment through mathematical modelling, it is necessary to obtain baseline condom use rates. Here, 
we present a novel method for estimation of such rates. In the absence of survey data, one possible method for assessing preand post-Avahan condom use rates and trends among samples of FSWs comparable with those recruited into the IBBA surveys is to 'reconstruct' presurvey condom use trends using the survey data itself.

We present a retrospective analysis of condom use time trends among FSWs with occasional and regular clients, in the districts where cross-sectional surveys have been carried out, and where the data necessary for this analysis were collected. We then present time-trend analyses and compare trends in condom use prior to and following the start of Avahan.

\section{METHODS}

IBBA

Appropriate data from the IBBA cross-sectional surveys were available from 21 out of 25 districts where the surveys were carried out among FSWs in the four southern Indian states of Andhra Pradesh, Karnataka, Maharashtra and Tamil Nadu. ${ }^{10}$ Round 1 (2006) survey data were used for this analysis, except for districts in Karnataka, where round 2 data (2007/8) were used, since the relevant questions were not included in the round 1 questionnaire for these districts. Target sample size was 400 per group; actual sample size varied from 153 to 422 per district ${ }^{4}$; and the total sample size for this study was 7358

\section{Reconstruction of retrospective condom use rates}

FSWs reporting consistent condom use (CCU) with occasional or regular clients were asked for how long they had used condoms consistently. Data on length of time consistently using condoms, and length of time selling sex, were converted into number of FSWs consistently using condoms (numerator) and selling sex (denominator) by year, to give yearly rates of CCU from 2001 to the year of the survey (2006 for most districts, 2007 for Mysore and 2008 for other Karnataka districts).

We use data from the Chennai IBBA (2006) as an example. Of a total of 348 FSWs who responded to the question: 'How often do you use condoms with your occasional clients?' 91\% (317) responded 'always.' The length of time these 317 sex workers had been using condoms consistently with clients was converted into years. Of the 317 FSWs using condoms consistently at the time of the survey (2006), 41 reported that they had been using condoms consistently for less than 1 year; thus, $((317-41)=276)$ had been using them for at least 1 year, that is, in 2005 . This calculation was repeated to give numbers using condoms consistently back to 2001 (table 1).

The same process was carried out to calculate the number of sex workers practising sex work by year. For example, of the sex workers interviewed, 13 reported that they had been selling sex for less than 1 year, and 39 for between 1 and 2 years: thus, 52 for less than 2 years. Therefore, $((348-13)=334)$ and $((348-52)=$ 296) sex workers were practising sex work in 2005 and 2004, respectively (table 1 ).

Then, to obtain the proportion of women consistently using condoms by year, the number consistently using condoms was divided by the number practising sex work in that year (table 1). For example, in Chennai in 2005, $((276 / 335)=82.4 \%)$ of sex workers selling sex in that year were using condoms consistently. These proportions were plotted on graphs with 95\% CIs (figure 1).

\section{Time-trend analysis}

Using these data, a generalised estimating equation (GEE) linear regression time-trend analysis, ${ }^{11}$ taking into account the sample
Table 1 Calculation of retrospective consistent condom use rates by female sex workers (FSWs) with occasional clients in Chennai, integrated biological and behavioural assessments cross-sectional survey 2006

Numerator: no of FSWs consistently using condoms by year

\begin{tabular}{lccllll}
\hline No years & No using & Cumul. & No using condoms for: & $\rightarrow$ No: & $\begin{array}{l}\rightarrow \text { No using } \\
\text { condoms in: }\end{array}$ \\
\hline $0(<1)$ & 41 & 41 & $\geq 1$ years $(317-41)$ & 276 & 2005 \\
$1(<2)$ & 136 & 177 & $\geq 2$ years $(317-177)$ & 140 & 2004 \\
$2(<3)$ & 70 & 247 & $\geq 3$ years $(317-247)$ & 70 & 2003 \\
$3(<4)$ & 38 & 285 & $\geq 4$ years $(317-285)$ & 32 & 2002 \\
$4(<5)$ & 16 & 301 & $\geq 5$ years $(317-301)$ & 16 & 2001 \\
Current & 317 & - & Currently: & 317 & 2006 \\
\hline
\end{tabular}

Denominator: no of FSWs practising sex work each year

\begin{tabular}{llcllll}
\hline No years & No sex work & Cumul. & No doing sex work for: & $\rightarrow$ No: & $\begin{array}{c}\rightarrow \text { No doing } \\
\text { sex work in: }\end{array}$ \\
\hline $0(<1)$ & 13 & 13 & $\geq 1$ years $(348-13)$ & 335 & 2005 \\
$1(<2)$ & 39 & 52 & $\geq 2$ years $(348-52)$ & 296 & 2004 \\
$2(<3)$ & 76 & 128 & $\geq 3$ years $(348-128)$ & 220 & 2003 \\
$3(<4)$ & 45 & 173 & $\geq 4$ years $(348-173)$ & 175 & 2002 \\
$4(<5)$ & 39 & 212 & $\geq 5$ years $(348-212)$ & 136 & 2001 \\
& & & & & \\
Current & 348 & - & Currently: & 348 & 2006
\end{tabular}

Proportion of FSWs using condoms consistently by year

\begin{tabular}{llll}
\hline Year & No using condoms & No doing sex work & $\begin{array}{l}\text { Percentage } \\
\text { using condoms }\end{array}$ \\
\hline 2001 & 16 & 136 & 11.8 \\
2002 & 32 & 175 & 18.3 \\
2003 & 70 & 220 & 31.8 \\
2004 & 140 & 296 & 47.3 \\
2005 & 276 & 335 & 82.4 \\
2006 & 317 & 348 & 91.1 \\
\hline
\end{tabular}

weights, the cluster effect and the dependency of the measures over time, was used to determine, for each district and for the whole sample:

a. the slopes of $\mathrm{CCU}$ increase, that is, the average yearly rate of increase in CCU from 2001 to year of survey;

b. the slopes of the pre- and post-Avahan CCU increases, that is the average yearly rate of increase in CCU prior to, and following, initiation of the Avahan intervention in 2003;

c. the difference between the pre- and postintervention slopes (ie, the difference in the average yearly rates of increase in CCU rates prior to, and following, implementation of the Avahan intervention).

The following hypotheses were then tested using $\chi^{2}$ tests:

a. that the overall slope was significantly different from zero (ie, that the overall upward trend in CCU was significant);

b. that the pre-Avahan intervention slope was significantly different from zero (ie, that the preintervention upward trend in CCU was significant);

c. that the post-Avahan intervention slope was significantly different from zero (ie, that the postintervention upward trend in CCU was significant);

d. that the pre- and postintervention slopes were significantly different (ie, that there was a difference in pre- and postAvahan intervention CCU trends).

For these analyses, the 'inflection point' was taken as 2003: that is, 2003 was considered as the last data point prior to the initiation of the Avahan programme; and 2004 was considered as the first data point after the initiation of the Avahan programme. The same year of initiation of the Avahan programme was used for all districts to keep the analysis simple and uniform, and to 


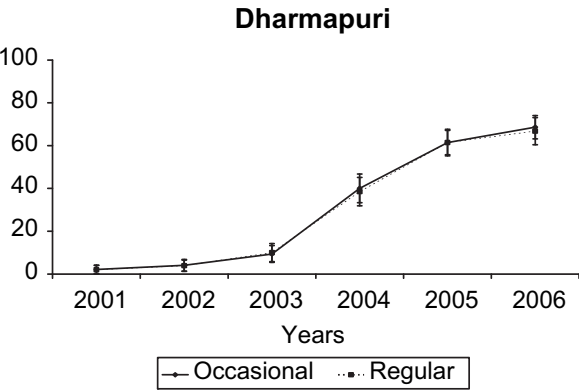

Visakhapatnam

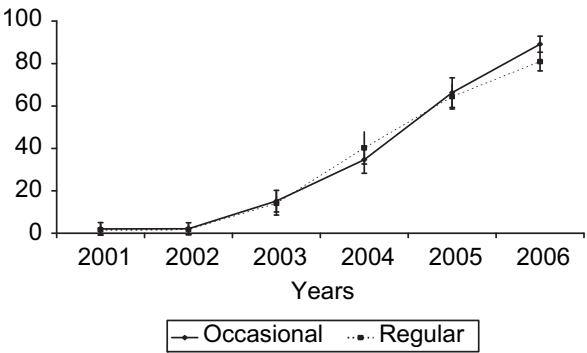

Mumbai NBB

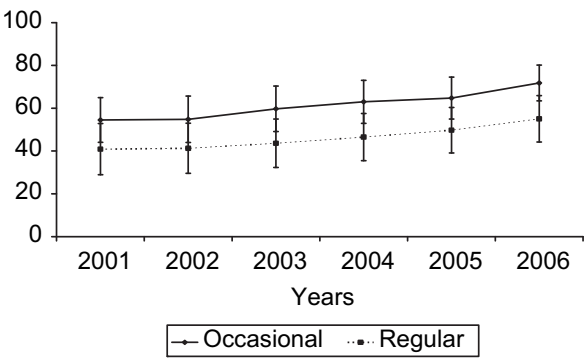

Guntur

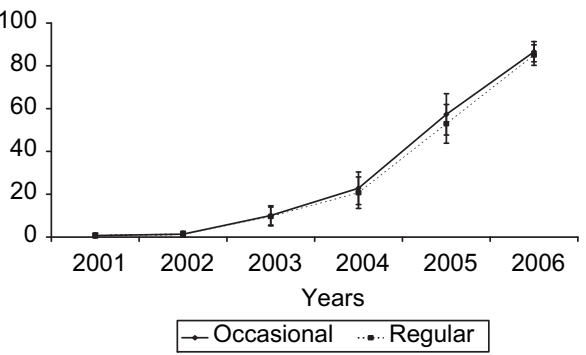

Bellary

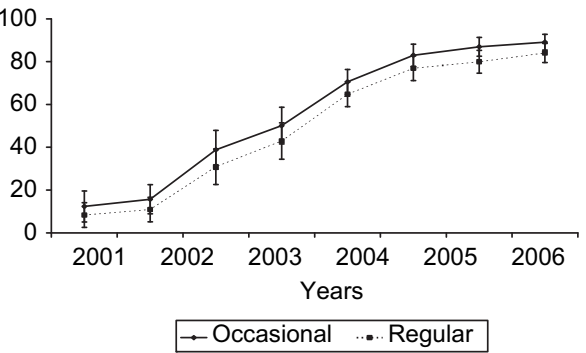

Pune BB

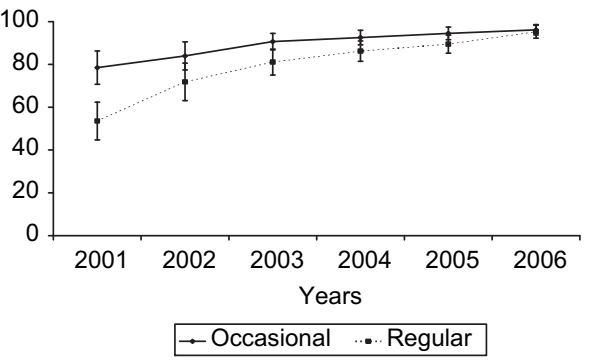

Mysore

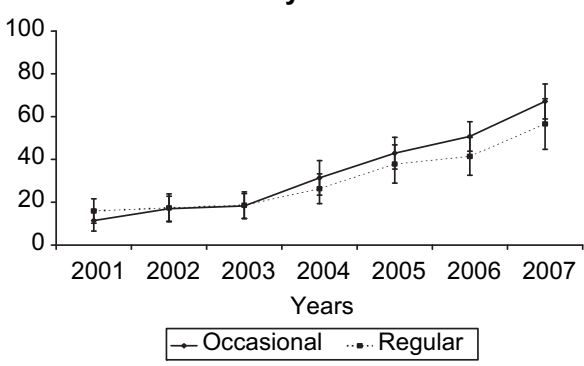

East Godavari

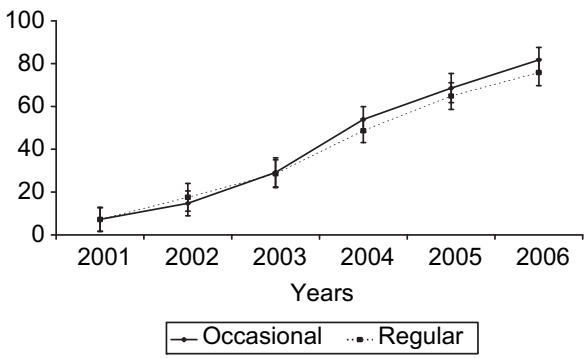

All districts combined

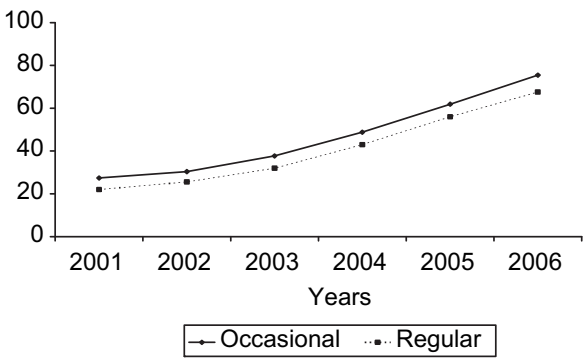

Figure 1 Reconstructed consistent condom use time trends by female sex workers with occasional and regular clients (2001 to year of survey), selected districts. NBB, Non brothel-based; BB, brothel-based.

avoid bias in assigning the inflection point visually. The year 2001 was used as the start year for the analysis in order to provide a sufficient number of preintervention years (three) for the timetrend analysis, while maintaining a sufficient sample size for the denominator of women practising sex work (table 1).

\section{Validation}

For districts in Karnataka (Belgaum, Bellary, Mysore, Shimoga), two rounds of IBBA survey data are available (University of Manitoba and Karnataka Health Promotion Trust. IBBA among FSWs in selected districts, Karnataka India: A Report, January 2008, KHPT, Bangalore (unpublished report)) and the retrospective analysis was carried out using the second-round data. For these districts, therefore, we were able to compare actual survey data on consistent condom use rates by FSW from survey round 1 with the results of the retrospective analysis for the same year the round 1 surveys were carried out.

\section{RESULTS}

Figure 1 shows graphs of reconstructed time trends in consistent condom use among sex workers with their occasional and regular clients, from 2001 (reconstructed data) to the year of the IBBA survey (survey data), for a selection of districts representing the main trend patterns identified, and for all districts combined (see Web Appendix for graphs of all 21 districts). Table 2, left-hand portion, shows CCU rates for 2001 and 2003 (reconstructed rates) and year of IBBA (survey data) for all districts. Table 2 also shows the state in which each of the districts is situated.

Striking differences among districts are seen in consistent condom use rates reported in the IBBA surveys, in reconstructed 2001 rates and in patterns of increase over time. Reconstructed 2001 rates vary from $0 \%$ with both regular and occasional clients in Chitoor (Andhra Pradesh), to 81\% with occasional clients in Thane (Maharashtra) non-brothel-based sex workers. IBBA survey (2006-2008) rates vary from $14.9 \%$ with regular clients in Chitoor, to $99 \%$ with occasional clients in Thane brothel-based sex workers.

In cases where 2001 rates are low, steep increases in condom use rates over time are seen-for example, in Bellary, Dharmapuri, East Godavari, Guntur, Mysore and Visakhapatnam (figure 1). 'Inflection points' - points (years) at which the slope of the curve changes quite sharply-can clearly be seen in figure 1, for Bellary and Visakhapatnam in 2002, for Dharmapuri and Mysore in 2003, and for Guntur in 2004. In some cases, such as in Bellary and Dharmapuri, rates of increase begin to level off in more recent years, from 2005/2006 onwards, whereas in other cases, such as Guntur and Visakhapatnam, rates appear to still be increasing quite steeply. In other districts-exemplified by Mumbai and Pune in figure 1-2001 rates were already relatively high, and relatively small increases over time are seen, with quite a flat curve. IBBA rates of condom use with occasional and regular clients tend to be similar, and reconstructed rates to follow similar patterns, with somewhat lower rates for regular clients (figure 1, table 2, left-hand portion). 
Table 2 Retrospective consistent condom use trends and linear time-trend analysis by district

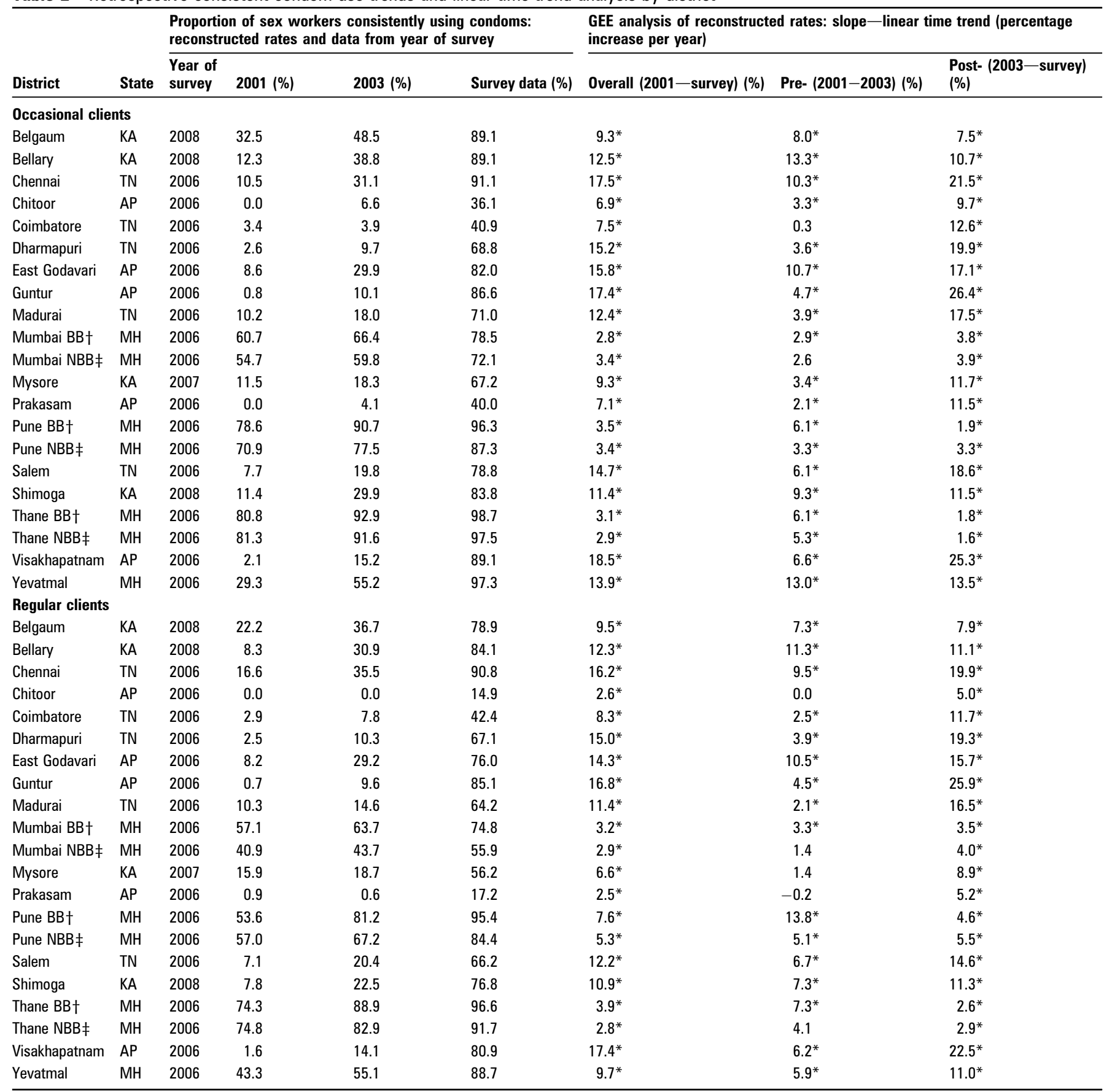

${ }^{*} \mathrm{p}<0.05$ ( $\chi^{2}$ test).

$+\mathrm{BB}=$ brothel-based.

$\ddagger N B B=$ non brothel-based.

AP, Andhra Pradesh; KA, Karnataka; MH, Maharashtra; TN, Tamil Nadu.

Combining data for all districts together, overall consistent condom use increased from $27 \%(22 \%)$ with occasional (regular) clients in 2001, to $76 \%(68 \%)$ in $2006(p<0.0001$, $\chi^{2}$ test).

As discussed above, while in some cases the graphs in figure 1 and Web Appendix figure 1 show clear 'inflection points,' in other cases, this is not clear. In order to provide an objective measure of the rate of increase in consistent condom use rates pre- and postAvahan implementation, we used GEE linear regression to determine the slope of condom use increase overall and pre- and post-Avahan, and to assess if there was a significant difference in rates of increase before and after the implementation of Avahan.
For these analyses, we defined 2003 as the last data point prior to Avahan, and 2004 as the first data point after Avahan initiation, in all districts, regardless of whether or in which year there was a clear visual inflection point on the graph.

Table 2 (right-hand portion) shows the results of the timetrend analysis-average percentage increase per year in consistent condom use rates overall, and pre- and postintervention, with results of significance testing for the overall upward trend, and the pre- and postintervention upward trends.

In all 21 districts, there was a highly significant $(p<0.001)$ overall upward trend (increase) in consistent condom use rates with occasional and regular clients from 2001 onwards (table 2, 
right-hand portion, column 'overall'). Yearly rates of increase range from $2.5 \%$ with regular clients in Prakasam (Andhra Pradesh) to $17.5 \%$ with occasional clients in Chennai (Tamil Nadu).

The pre-Avahan (2001-2003) upward trend in condom use rates with occasional and regular clients was significant in 19/21 and 16/21 districts respectively, while the post-Avahan (2003-2006) upward trend was significant in all 21 districts, for both occasional and regular clients. Preintervention average yearly rates of increase were lowest among FSWs with regular clients in Prakasam (Andhra Pradesh) at $-0.2 \%$; and highest among brothel-based sex workers with regular clients in Pune (Maharashtra), at $13.8 \%$ per year. Postintervention average yearly rates of increase varied from $2.6 \%$ among non-brothel based sex workers with regular clients in Thane (Maharashtra), to $26 \%$ among FSWs with occasional clients in Guntur (Andhra Pradesh).

In order to provide a summary measure to compare increases in consistent condom use rates before and after the initiation of the Avahan programme, the difference between the preintervention and postintervention average yearly rates of increase was calculated for each district, and the statistical significance of the difference between the rates assessed (table 3).

Differences in yearly rates of pre- and postintervention increase varied from $-9.2 \%$ to $+21.7 \%$. This means, for example, that in the case of condom use by brothel-based sex workers with regular clients in Pune (Maharashtra), the average yearly increase in rates was about $9 \%$ greater before the start of Avahan than after it (negative inflection point). Conversely, in the case of condom use by sex workers with occasional clients in Guntur (Andhra Pradesh), the average yearly increase in rates was about $22 \%$ greater after the start of Avahan than before it (positive

Table 3 Retrospective analysis of condom use trends: summary: comparison of pre- and postintervention rates of CCU increase (slopes) using linear regression with GEE, by district

\begin{tabular}{|c|c|c|c|c|c|}
\hline \multirow[b]{2}{*}{ District } & \multirow[b]{2}{*}{ State } & \multicolumn{2}{|c|}{ Occasional clients } & \multicolumn{2}{|c|}{ Regular clients } \\
\hline & & $A(\%)$ & B & A (\%) & B \\
\hline Belgaum & KA & -0.5 & $=$ & 0.7 & $=$ \\
\hline Bellary & KA & -2.6 & $=$ & -0.2 & $=$ \\
\hline Chennai & TN & $11.2^{*}$ & + & $10.5^{*}$ & + \\
\hline Chitoor & AP & $6.4^{*}$ & + & $5.0^{*}$ & + \\
\hline Coimbatore & TN & $12.3^{*}$ & + & $9.3^{*}$ & + \\
\hline Dharmapuri & TN & $16.3^{*}$ & + & $15.4^{*}$ & + \\
\hline East Godavari & AP & 6.4 & $=$ & 5.2 & $=$ \\
\hline Guntur & AP & $21.7^{*}$ & + & $21.4^{*}$ & + \\
\hline Madurai & TN & $13.6^{*}$ & + & $14.4^{*}$ & + \\
\hline Mumbai BB† & $\mathrm{MH}$ & 0.9 & $=$ & 0.3 & $=$ \\
\hline Mumbai NBB $\ddagger$ & $\mathrm{MH}$ & 1.3 & $=$ & 2.6 & $=$ \\
\hline Mysore & KA & $8.3^{*}$ & + & $7.5^{*}$ & + \\
\hline Prakasam & AP & $9.4^{*}$ & + & $5.3^{*}$ & + \\
\hline Pune BB† & $\mathrm{MH}$ & $-4.2^{*}$ & - & $-9.2^{*}$ & - \\
\hline Pune NBB $\ddagger$ & $\mathrm{MH}$ & 0 & $=$ & 0.4 & $=$ \\
\hline Salem & TN & $12.5^{*}$ & + & $8.0^{*}$ & + \\
\hline Shimoga & $\mathrm{KA}$ & 2.2 & $=$ & 4.0 & $=$ \\
\hline Thane BB† & $\mathrm{MH}$ & $-4.3^{*}$ & - & $-4.7^{*}$ & - \\
\hline Thane NBB $\ddagger$ & $\mathrm{MH}$ & -3.7 & $=$ & -1.2 & $=$ \\
\hline Visakhapatnam & AP & $18.8^{*}$ & + & $16.2^{*}$ & + \\
\hline Yevatmal & $\mathrm{MH}$ & 0.6 & $=$ & 5.1 & $=$ \\
\hline
\end{tabular}

Column $A$, absolute difference in average yearly rate of increase in CCU rates (slope); column B, type of inflection point: + , positive inflection point: post- significantly greater than pre- rate of increase; $=$, no inflection point: no significant difference between post- and pre- rates of increase; -, negative inflection point: pre- significantly greater than post- rate of increase. AP, Andhra Pradesh; KA, Karnataka; MH, Maharashtra; TN, Tamil Nadu.

${ }^{*} \mathrm{p}<0.05$ ( $\chi^{2}$ test).

$+\mathrm{BB}=$ brothel-based

$\ddagger \mathrm{NBB}=$ non brothel-based inflection point). In a number of districts, the pre-post difference in rates was not significant, indicating that the yearly rate of increase in condom use was not different after than before initiation of Avahan (no inflection point).

To summarise these results: in the case of both regular and occasional clients, 10/21 districts had a positive inflection point, 9/21 had no inflection point, and 2/21 had a negative inflection point. In districts with significantly different post- and preintervention slopes $(n=12)$, the number of districts with a positive inflection point $(n=10)$ was significantly greater than the number with a negative inflection point $(n=2)(p=0.039)$, using the $\mathrm{McNemar} \chi^{2}$ test.

Combining data from all districts, the yearly rate of increase in consistent condom use was significantly greater following than prior to Avahan (positive inflection point). Thus, the 2003-2006 average yearly rates of increase were $12.7 \%$ (12.0\%) per year for occasional (regular) clients; compared with the 2001-2003 rates of 5.1\% (5.3\%) per year for occasional (regular) clients $\left(p<0.0001, \chi^{2}\right.$ test).

Finally, a comparison of consistent condom use rates with clients actually reported by FSW in the first round of IBBA surveys carried out in 2004/2005, with rates calculated retrospectively for that year using second round 2007/2008 survey data, is shown in table 4. Seven comparison points are available. The point estimates differ by between $0 \%$ and $10.2 \%$. For six out of seven comparisons - that is, all apart from Belgaum occasional clients-the overlap between the $95 \%$ CIs in each case indicates that there is no significant difference between the actual and reconstructed estimates.

\section{DISCUSSION}

Using a retrospective analysis of IBBA survey data from 21 districts in southern India, collected within the context of an evaluation of the impact of the Avahan programme, we 'reconstructed' historical consistent condom use trends among FSWs with their occasional and regular clients from 2001 onwards, and analysed the relationship between these trends and the initiation of the Avahan programme in 2003.

Table 4 Consistent condom use rates by female sex workers with clients: comparison of reported survey rates with 'reconstructed' rates: Karnataka state

\section{Mysore 2004}

Survey: regular clients

Percentage $(95 \% \mathrm{Cl})$

Reconstructed: regular clients 20.0 (10.9 to 29.2 ) 26.5 (19.9 to 33.1)

\section{Belgaum 2005}

Survey: occasional clients

Reconstructed: occasional clients

Survey: regular clients

Reconstructed: regular clients

90.9 (87.4 to 94.3$)$ 80.7 (75.9 to 85.5$)$ 70.1 (59.6 to 80.6$)$ 70.1 (64.6 to 75.6$)$

\section{Bellary 2005}

Survey: occasional clients

Reconstructed: occasional clients

Survey: regular clients

Reconstructed: regular clients

72.6 (66.7 to 78.4$)$

70.6 (64.7 to 76.4$)$

55.5 (42.7 to 68.3$)$

64.8 (59.0 to 70.6$)$

\section{Shimoga 2005}

Survey: occasional clients

54.5 (48.1 to 60.7 )

Reconstructed: occasional clients

Survey: regular clients

Reconstructed: regular clients 
The analyses indicate that significant increases in condom use rates occurred from 2001 to the year of survey (2006-2008) in all 21 districts for which data were available. Pre-2003 yearly rates of increase were significant in 19 (occasional clients) and 16 (regular clients) districts, while post-2003 rates of increase were significant in all 21 districts for both occasional and regular clients. There were significantly more districts where the post-2003 rate of increase was greater than the pre-2003 rate, compared with the converse (ie, pre- greater than post-2003 rate), for both regular and occasional clients.

Combining all districts, consistent condom use rates with occasional clients increased from $27 \%$ in 2001 , to $76 \%$ in 2006 ; and with regular clients, from $22 \%$ to $68 \%$. The yearly rate of increase in consistent condom use for all districts combined was significantly greater following than prior to Avahan initiation.

These findings indicate a positive relationship between the time of implementation of the Avahan programme and rates of increase in consistent condom use among FSWs. Taken together, the data indicate that (a) condom use rates were already on the increase (to varying extents and from different 'baseline' (2001) rates, according to district) before the implementation of Avahan; and (b) the yearly rate of increase in consistent condom use accelerated further at a time coincident with the implementation of the Avahan intervention.

As presented above (figure 1), striking differences among districts were seen in condom use rates reported in the IBBA surveys, in reconstructed 2001 rates and in patterns of increase over time. These differences are likely related at least in part to the timing of introduction and coverage of pre-Avahan interventions. In Maharashtra state, for example (Mumbai, Pune and Thane districts), where in general interventions with FSWs commenced relatively early on, these data suggest that consistent condom use rates were already high in 2001 - ranging from 50 to $80 \%$; subsequent increases were small, and post-Avahan yearly rates of increase were equal to, or lower than, pre-Avahan rates of increase. In contrast, in districts where pre-Avahan FSW interventions had not been implemented, started later or had lower coverage (districts in Karnataka; some districts in Andhra Pradesh and Tamil Nadu), 2001 rates were lower, and post-Avahan yearly rates of increase were greater than preAvahan rates.

The retrospective analysis technique depends on the accuracy of the survey data used as a starting-point. In a situation such as this, where intensive targeted interventions are under way, covering significant proportions of the FSW population, social desirability bias ${ }^{12}$ is likely to be high, and self-reported condom use rates inflated. ${ }^{13-15}$ Social desirability bias could also be at play when FSW recount the length of time they have been using condoms, for example influencing their responses in terms of the reported length of time using condoms consistently. However, the quite striking variation in 2001 rates and in patterns of increase over time (eg, that reconstructed rates of consistent condom use in Chitoor were $0 \%$ in 2001, compared with $81 \%$ in Thane) gives some confidence that at least FSW are reporting consistent condom use for significantly varying periods of time.

There are some additional limitations to this analysis. An additional potential source of bias is recall bias-both of the length of time using condoms and of practising sex work. ${ }^{10}$ Furthermore, only sex workers practising sex work at the time of the survey are included. For example, the 2003 'retrospective' sample would not include those sex workers who were practising sex work in 2003 but who gave up in 2004, 2005 or 2006, as would be included if survey data were available from 2003. This may result in a tendency towards a longer average duration of sex work among the retrospective compared with the actual sample in any given year. In addition, the sample size becomes progressively smaller as the years go back, since not all women practising sex work in 2006 will have been doing so in 2005, and so on. For example, in Chennai (table 1), of 348 women interviewed in 2007,136 (39\%) reported practising sex work in 2001. It is worth noting that we repeated the time-trend analyses presented here controlling for duration of sex work, and this did not affect the results (data not shown), suggesting that the tendency towards a longer average duration of sex work in the retrospective sample does not result in a systematic bias.

While in most Avahan IBBA districts, only one round of IBBA has so far been carried out (2006), in the districts in Karnataka state, two rounds of IBBA have been conducted, ${ }^{7}$ and the second-round IBBA surveys (2007/8) were used for the retrospective analysis. We were thus able to compare consistent condom use rates estimated retrospectively with rates actually reported in surveys for the same year; we found in six out of seven comparisons that there was no significant difference between the actual and the reconstructed estimates, as indicated by the overlap between the $95 \%$ CIs in each case (table 4). As data from further survey rounds become available, it will be possible to carry out more extensive comparisons and validations; there may also be survey datasets available from other projects, with the appropriate questions, that could be used to examine the validity of the method.

The results of our analysis are also supported by those of Bradley et $a l,{ }^{16}$ who used data on condom availability in five districts in Karnataka (four of which were the same as the IBBA survey districts presented here) from governmental and nongovernmental sources, surveys and HIV-prevention programmes, to estimate the number of available condoms by year from 2004 to 2008, and to estimate possible condom use rates among FSW under a number of different scenarios. This analysis suggests that condom availability was relatively low in Karnataka in 2004 but rose substantially over the following 4 years, as did estimated condom use rates by FSW, from $3-36 \%$ in 2004 to $56-96 \%$ in $2008 .{ }^{16}$ This is in agreement with the results of the analysis presented here, which also demonstrate very substantial increases in condom availability and use during this period.

In conclusion, the findings presented here indicate a positive relationship between the time of implementation of the Avahan

\section{Key messages}

- We present a novel method for estimation of historical condom use rates among female sex workers in India, using crosssectional survey data on duration of condom use and duration of sex work.

- Comparison of reconstructed condom use rates with actual data collected in the same year suggests that the method is valid.

- The method could be applied in other contexts to estimate preintervention condom use rates for programme evaluation in the absence of baseline data. We recommend the inclusion of questions on duration of behaviours in programme evaluation surveys.

- The findings of the analysis indicate a positive relationship between implementation of the Avahan programme and rates of increase in consistent condom use among female sex workers. 
programme and rates of increase in consistent condom use among FSWs. This is in agreement with data indicating that the Avahan intervention has achieved a high coverage of sex worker populations in southern India, and a number of lines of evidence indicating that the programme is having a positive impact on increasing condom use rates, and decreasing STI rates and numbers of new HIV infections among the target population. 78 17-18

Further validation of the methodology and the findings of this analysis will be carried out as more data become available. We recommend that future surveys carried out in the context of programme evaluations could include the specific questions necessary to carry out retrospective analysis, namely duration of condom use and duration of sex work. This analytical method may thus be of utility in other contexts where comparable baseline/preintervention data are lacking for assessment of changes over time in behaviour and the outcome of interventions.

Acknowledgements This research was funded by the Bill \& Melinda Gates Foundation. The views expressed herein are those of the authors and do not necessarily reflect the official policy or position of the Bill \& Melinda Gates Foundation. MA is a national researcher of the Fonds de la Recherche en Santé du Québec, Canada (grant no. 8722).

Contributors CML developed the idea for the study and wrote the manuscript. MA is $\mathrm{Pl}$ of the CHARME-India Project; he supervised the data and statistical analyses and assisted with writing and revision of the manuscript. SV, ED and AAJ carried out data and statistical analyses, and reviewed the manuscript. JB managed the data analysis and reviewed the manuscript. BMR, SM, RA and MKM were responsible for survey data collection and reviewed the manuscript.

Funding Bill and Melinda Gates Foundation, P0 Box 23350, Seattle, WA 98102, USA. Competing interests None.

Ethics approval Ethics approval was provided by the Centre hospitalier affilié universitaire de Québec, Québec, Canada; University of Manitoba, Winnipeg, Canada; St John's Medical College, Bangalore, India.

Provenance and peer review Not commissioned; externally peer reviewed.

\section{REFERENCES}

1. Avahan. The India AIDS Initiative. The business of HIV prevention at scale. New Delhi (India): Bill \& Melinda Gates Founation, 2008. http://www.gatesfoundation.org/ avahan/Documents/Avahan HIVPrevention.pdf.

2. Chandrasekaran P, Dallabētta G, Loo V, et al; Avahan Evaluation Partners. Evaluation design for large-scale HIV prevention programmes: the case of Avahan, the India AIDS Initiative. AIDS 2008:22(Suppl 5):S1-15.

3. Boily MC, Lowndes CM, Vickerman P, et al; CHARME-India team. Evaluating largescale HIV prevention interventions: study design for an integrated mathematical modelling approach. Sex Transm Infect 2007;83:582-9.
4. Saidel T, Adhikary R, Mainkar M, et al. Baseline integrated behavioural and biological assessment among most at-risk populations in six high-prevalence states of India: design and implementation challenges. AIDS 2008;22(Suppl 5): S17-34.

5. Williams JR, Foss AM, Vickerman P, et al. What is the achievable effectiveness of the India AIDS Initiative intervention among female sex workers under target coverage? Model projections from southern India. Sex Transm Infect 2006;82:372-80

6. Pickles $\mathbf{M}$, Foss $A M$, Vickerman $\mathbf{P}$, et al. Interim modelling analysis to validate reported increases in condom use and assess HIV infections averted among female sex workers and clients in southern India following a targeted HIV prevention programme. Sex Transm Infect 2010;86(Suppl 1):i33-43.

7. Ramesh BM, Beattie TSH, Shajy I, et al. Changes in risk behaviours and prevalence of sexually transmitted infections following HIV preventive interventions among female sex workers in five districts in Karnataka State, south India. Sex Transm Infect 2010;86(Suppl 1):i17-24.

8. Verma R, Shekhar A, Khobragade S, et al. Scale-up and coverage of Avahan: a large scale HIV-prevention programme among female sex workers and men who have sex with men in four Indian states. Sex Transm Infect 2010;86(Suppl 1): i76-82.

9. Fung IC, Guinness L, Vickerman P, et al. Modelling the impact and cost-effectiveness of the HIV intervention programme amongst commercial sex workers in Ahmedabad Gujarat, India. BMC Public Health 2007; 7:195.

10. Indian Council of Medical Research \& Family Health International. National Interim Summary Report (October 2007), Integrated Behavioural and Biological Assessment (IBBA), Round 1 (2005-2007).

11. Hanley JA, Negassa A, Edwardes MD, et al. Statistical analysis of correlated data using Generalized Estimating Equations: an orientation. Am J Epidemiol 2003;157:364-75

12. Catania JA, Gibson DR, Chitwood DD, et al. Methodological problems in AIDS behavioral research: influences on measurement error and participation bias in studies of sexual behavior. Psychol Bull 1990;108:339-62.

13. Hanck SE, Blankenship KM, Irwin KS, et al. Assessment of self-reported sexual behavior and condom use among female sex workers in India using a polling box approach: a preliminary report. Sex Transm Dis 2008;35:489-94.

14. Thomsen SC, Gallo MF, Ombidi W, et al. Randomised controlled trial on whether advance knowledge of prostate-specific antigen testing improves participant reporting of unprotected sex. Sex Transm Infect 2007;83:419-20.

15. Zenilman JM, Weisman CS, Rompalo AM, et al. Condom use to prevent incident STDs: the validity of self-reported condom use. Sex Transm Dis 1995:22:15-21.

16. Bradley J, Moses S, Blanchard JF, et al. Assessing reported condom use among female sex workers in southern India through examination of condom availability. Sex Transm Infect 2010;86(Suppl 1):i44-8.

17. Reza-Paul S, Beattie T, Syed HU, et al. Declines in risk behaviour and sexually transmitted infection prevalence following a community-led HIV preventive intervention among female sex workers in Mysore, India. AIDS 2008;22(Suppl 5): S91-100.

18. Pickles $\mathbf{M}$, Foss AM, Vickerman $\mathrm{P}$, et al. Interim modelling analysis to validate reported increases in condom use and assess HIV infections averted among female sex workers and clients in southern India following a targeted HIV prevention programme. Sex Transm Infect 2010;86:i33-43.

19. Ramakrishnan L, Gautam A, Goswami P, et al. Programme coverage, condom use and STI treatment among FSWs in a large-scale HIV prevention programme: results from cross-sectional surveys in 22 districts in southern India. Sex Transm Infect 2010;86:i62-8. 